\title{
Challenges in the Identification of New Thermolabile Psychoactive Substances: the 25I-NBOH case.
}

Ana Flávia Belchior de Andrade ${ }^{1}$, Mathieu Elie ${ }^{2}$, Christian Weck², Jorge Jardim Zacca ${ }^{3}$, Mônica Paulo de Souza ${ }^{3}$, Luíza Nicolau Brandão Caldas ${ }^{3}$, Jose Gonzalez-Rodriguez ${ }^{2}$

${ }^{1}$ Seção de Perícias e Análises Laboratoriais, Instituto de Criminalística, Polícia Civil do Distrito Federal, SPO, Lote 23, Bloco E, Brasília, DF CEP 70610-200, Brazil

${ }^{2}$ School of Chemistry, University of Lincoln, Brayford Pool, Lincoln LN6 7TS, UK

${ }^{3}$ Instituto Nacional de Criminalística, Polícia Federal, SPO - Lote 7 - Setores Complementares Brasília/DF - CEP 70.610-902, Brazil

\section{Highlights}

1. Thermolabile NPS can generate artefacts in the traditional GC-MS analysis

2. When analysed by GC, 25I-NBOH fragments into $2 \mathrm{C}-\mathrm{I}$ and an ortho-phenolic benzyl ether (o-PBE)

3. No method adjustments can refrain $25 \mathrm{I}-\mathrm{NBOH}$ thermo degradation on $\mathrm{GC}$ analysis

4. DSC and TGA analysis clarify $25 \mathrm{I}-\mathrm{NBOH}^{\prime} \mathrm{s}$ thermal instability

5. Derivatization can overcome 25I-NBOH degradation in the GC-MS

\begin{abstract}
The continuous emergence of NPS over the last years poses a series of novel challenges for forensic analysts. Most of those new compounds are synthesized with minimal chemical modifications to the structure of already known chemicals in order to avoid regulations. Some of these new compounds may undergo chemical changes during analysis leading to misidentification and detrimental legal consequences. GC-MS is one of the most widely used analytical techniques employed by forensic laboratories all over the world for drug analysis. Nevertheless, thermolabile NPS, such as $25 \mathrm{I}-\mathrm{NBOH}$ can generate artefacts in the traditional GCMS analysis. In this paper, we describe the fragmentation mechanism of the $25 \mathrm{I}-\mathrm{NBOH}$ into a major peak corresponding to $2 \mathrm{C}-\mathrm{I}$ and a minor one corresponding to the associated orthophenolic benzyl ether (O-PBE), which exact identity is directly linked with the solvent used for the analysis. Also, a series of method adjustments is displayed, encompassing variation on the injector temperature, split ratio and flow ratio, although with no success to prevent 25I-NBOH thermo degradation in the GC injector. Furthermore, differential scanning calorimetry and thermogravimetric analysis demonstrated that $25 \mathrm{I}-\mathrm{NBOH}^{\prime} \mathrm{s}$ thermal stability is due to a smaller temperature window between fusion and decomposition points. Finally, we perform derivatization experiments and demonstrate how to overcome $25 \mathrm{I}-\mathrm{NBOH}$ degradation in the GC/MS analysis.
\end{abstract}

Key words 25I-NBOH, NPS, thermal degradation, derivatization 


\section{Introduction}

In the last decade, novel psychoactive substances (NPS) have transformed the global synthetic drug market [1]. Recent advances in organic chemistry have allowed the low cost and easy to replicate synthesis of hundreds of different substances with the desired chemical functionality as to emulate target psychoactive effects in the human body. As a consequence an unprecedented proliferation of NPS, in terms of both quantity and diversity has been witnessed [2]. Moreover, trends on the synthetic drug market quickly evolve each year complicating the challenges to monitor, understand and control of synthetic drugs and their chemicals precursors [3].

One important category of NPS are thermolabile compounds, which are not prone to degrade under typical gas chromatography injection conditions due to the occurrence of strong intramolecular hydrogen bonds. This type of substance will experience thermal degradation and it is not likely to be identified by routine gas chromatographic methods. Among them, the substituted psychedelic phenethylamine $25 \mathrm{I}-\mathrm{NBOH}$ has become a benchmark case right after NBOMes have been scheduled [4].

An understanding of the thermal behavior of $25 \mathrm{I}-\mathrm{NBOH}$ is crucial to determine whether degradation can be avoided in GC analysis. Differential scanning calorimetry (DSC) is frequently the preferred thermal analytical technique used because of its ability to provide detailed information regarding both, physical and energetic properties of a substance [5]. This technique provides quantitative information about exothermic, endothermic and heat capacity changes as a function of temperature and determines whether there are underlying slow degradation or decomposition processes occurring [6]. In this context Thermogavimetric Analisys (TGA) also delivery critical information by measuring changes in the physico-chemical properties at elevated temperature as a function of increasing temperature. Therefore essential data regarding stability, decomposition and compound structure can be clarified using TGA [7]. Both methodologies can provide key information to comprehend the degradation process on 25I$\mathrm{NBOH}$ compounds and be a useful tool to drive further actions in an attempt to prevent degradation.

Due to thermo instability, $\mathrm{GC}$ analyses of $25 \mathrm{I}-\mathrm{NBOH}$ will not be able to unequivocally establish if all the $2 \mathrm{C}-\mathrm{I}$ present in the chromatogram is due to $25 \mathrm{I}-\mathrm{NBOH}$ thermal degradation alone or whether $2 \mathrm{C}-\mathrm{I}$ was originally present in the mixture. This fact implies a serious legal problem as $25 \mathrm{I}-\mathrm{NBOH}$ is not a scheduled drug in many countries whereas $2 \mathrm{C}-\mathrm{I}$ is [8].

Although other analytical techniques such as differential pulse voltammetry (DPV), near-infrared spectroscopy (NIR) and easy ambient sonic-spray mass spectrometry imaging (EASI-IMS) can successfully overcome this problem [9-11], GC-MS is the most widely used technique for identification/confirmation of drugs by forensic experts. As an example, approximately $90 \%$ of forensic laboratories that participate in the International Collaborative exercise proposed by UNODC in 2018 utilized GC-MS as a reference method for drug analyses [12].

Consequently, the development of a improved GC method for the correct identification of 25I$\mathrm{NBOH}$ is of high relevance for forensic analysts all over the world. In this paper, we explore the fragmentation mechanism of the $25 \mathrm{I}-\mathrm{NBOH}$, present our efforts to avoid its thermo degradation 
in the GC injector, display thermal analysis results and suggest a solution to overcome the presented problem.

\section{Material and Method}

\section{Study samples}

25I-NBOMe and 25I-NBOH certified standard was purchased from Cayman Chemical (Ann Arbor, MI, USA). 2C-I certified standard was kindly donated by United Nations Office on Drugs and Crime (UNODC). Blotter papers containing 25I-NBOH were donated by Brazilian Federal Police. Methanol, N-Methyl-N-(trimethylsilyl) trifluoroacetamide (MSTFA), N,O-Bis(trimethylsilyl) trifluoroacetamide (BSTFA), acetic anhydride and pyridine were purchased from Merck KGaA (Darmstadt, Germany).

\section{Analysis of thermal behavior of 25I-NBOH using DSC and TGA}

Differential scanning calorimetry (DSC) of the 25I-NBOH was carried out using a differential scanning calorimeter DSC822e from Mettler-Toledo. For the analysis $2.53 \mathrm{mg}$ of $25 \mathrm{I}-\mathrm{NBOH}$ and about $2.33 \mathrm{mg}$ of $25 \mathrm{I}-\mathrm{NBOMe}$ were used. The calorimeter was programmed for a heating ramp between $30^{\circ} \mathrm{C}$ and $500^{\circ} \mathrm{C}$ with a rate of $10^{\circ} \mathrm{C} \mathrm{min}{ }^{-1}$. Measurements were performed under a Nitrogen (N2) atmosphere with a constant flux of $80 \mathrm{~mL} \mathrm{~min}^{-1}$.

Thermogravimetric analysis was conducted using a TGA/SDTA851e module (Mettler-Toledo, Columbus, OH, EUA). For the analysis $2.47 \mathrm{mg}$ of $25 \mathrm{I}-\mathrm{NBOH}$ and $2.22 \mathrm{mg}$ of 25I-NBOMe were used. A heating rate of $20^{\circ} \mathrm{C} \mathrm{min}^{-1}$ was programmed in the temperature range of $30^{\circ} \mathrm{C}$ to $800^{\circ} \mathrm{C}$

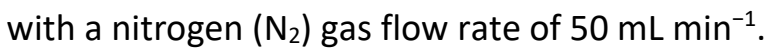

\section{Qualitative analysis of 25I-NBOH by GC-MS}

GC-MS analyses were performed on an Agilent 7890A gas chromatograph connected to a $5975 \mathrm{C}$ mass spectrometer (Agilent Technologies, Santa Clara, CA, USA). The system was controlled by Agilent Chemstation GC-MS Software version E 02.02.1431 (Agilent Technologies, Santa Clara, CA, USA). For the GC-MS analysis an Agilent J\&W HP-1MS fused silica capillary column ( $30 \mathrm{~m} x$ $0.25 \mathrm{~mm} \times 0.25 \mu \mathrm{m}$ ) was used. Sample injection volume was $1 \mu \mathrm{L}$ with a 25:1 split ratio. Helium was used as carrier gas, with a constant flow rate of $1 \mathrm{~mL} \mathrm{~min}^{-1}$. Injector temperature was set to $280^{\circ} \mathrm{C}$. The oven program started at $150^{\circ} \mathrm{C}$ with a hold for $1.5 \mathrm{~min}$, ramped up at $30^{\circ} \mathrm{C} \mathrm{min}^{-1}$ to reach $250^{\circ} \mathrm{C}$ with a hold for $1 \mathrm{~min}$, and then ramped up at $50^{\circ} \mathrm{C} \mathrm{min}^{-1}$ to $300^{\circ} \mathrm{C}$ with a hold for 3 $\mathrm{min}$. The transfer line temperature was set at $300^{\circ} \mathrm{C}$. The solvent delay was set to $1.5 \mathrm{~min}$. Mass scan range was $m / z$ 35-550.

\section{Derivatization procedure of 25I-NBOH}

Three derivatizing agents were tested: $\mathrm{N}-$ Methyl-N-(trimethylsilyl) trifluoroacetamide (MSTFA), $\mathrm{N}, \mathrm{O}-\mathrm{Bis}$ (trimethylsilyl) trifluoroacetamide (BSTFA) and acetic anhydride. For each one, five blotter paper micro stamps originated from Brazilian Federal Police seizures were immersed into $1.5 \mathrm{~mL}$ of methanol and extracted in an ultrasound bath for $30 \mathrm{~min}$. The extract was then dried to a powder in a rotary evaporator at room temperature. In the case of MSTFA and BSTFA, 150 $\mu \mathrm{L}$ were added in the dry extract, vortexed for $10 \mathrm{~s}$. In the case of acetic anhydride, $100 \mu \mathrm{L}$ of the 
derivatization reagent and $100 \mu \mathrm{L}$ of pyridine were added the dried extract before vortexing. In all cases, the resulting solutions were kept in a vial heater at $70^{\circ} \mathrm{C}$ for $1 \mathrm{~h} .1 \mu \mathrm{L}$ of the reconstituted solutions, without any additional solvent, was injected into the GC-MS.

\section{Results and Discussion}

\section{I-NBOH in-port degradation and proposed fragmentation of $o$-PBEs}

As previously reported [8], 25I-NBOH undergoes degradation during GC analyses. When injected in the presence of an alcohol, two peaks can be observed: a major one corresponding to $2 \mathrm{C}-\mathrm{I}$, and a minor one corresponding to the associated ortho-phenolic benzyl ether (o-PBE). The exact identity of the $O-P B E$ observed is directly linked with the solvent used for the analysis. We propose here the degradation mechanism and how it affects the resulting mass spectra of the different $o$-PBEs created.

When introduced in the injector, 25I-NBOH can be considered as a zwitterion solvated by excess alcohol in the gas phase. Under high temperature conditions $\left(280^{\circ} \mathrm{C}\right)$ and relatively high pressure in the liner environment, the alcohol acts as a nucleophilic group and the $2 \mathrm{C}-\mathrm{I}$ as a leaving group. The electron-rich oxygen from the alcohol replaces the 2C-I group to form an ether. As a zwitterion, the natural tendency of $25 \mathrm{I}-\mathrm{NBOH}$ to undergo an intra-molecular six-membered proton transfer, leading to a positive nitrogen and a negative oxygen on its phenolic moiety, enables the capturing of the hydrogen from the alcohol's hydroxyl and "facilitates" the nucleophilic substitution (Figure 1 (a)). This interpretation is supported by mass spectra obtained by Neto [8] where 25I-NBOH was solvated in tetradeuterated methanol.

Indeed, while the 2C-I fragmentation mechanism is classically based upon a series of cleaves, the $o$-PBEs presents a more complex picture (Figure 2). When using methanol, ethanol, and $n$ propanol as the solvent, molecular ions $[\mathrm{M}]^{+}$are observed (respectively $\mathrm{m} / \mathrm{z} 138,152$, and 166) as well as a series of identical daughter ions $(\mathrm{m} / \mathrm{z} 121,106,78$, and 51$)$.

Two mechanical pathways, each starting with an $\alpha$-cleavage on one side of the ether, allows explaining the presence of these ions (Figure 1 (b)). Pathway 1 considers the $\alpha$-cleavage of the ether moiety. The positively charged intermediate stabilizes its charge in the phenyl ring through tautomeric interconversions, followed by a hydrogen abstraction. It leads to the formation of oxo-tropylium ions $(\mathrm{m} / \mathrm{z} 106)$ via aromatic rearrangement, followed by the $\alpha$-cleavage of the carbonyl group producing stable phenylium ions $(\mathrm{m} / \mathrm{z} 78)$, and subsequent loss of acetylene ($\left.\mathrm{C}_{2} \mathrm{H}_{2}\right)$ creating cyclobutandienylium ions $(m / z 51)$ [13]. Such patterns have been previously reported for tropones and aromatic systems [14].

Pathway 2 considers the $\alpha$-cleavage of the alkyl tail of the ether. Two subsequent abstractions of hydrogen result in the production of [(2-hydroxyphenyl)methylidyne]oxonium ions $(\mathrm{m} / \mathrm{z} 121)$. The particularity of this ion is that it is the only daughter ion retaining the hydrogen originally provided by the solvent to the $o-\mathrm{PBE}$. Indeed, this is why the mass spectrum of the o-PBE obtained with tetradeuterated methanol [8] shows a $m / z 122$ ion, corresponding to the $m / z 121$ ion when using non-deuterated solvents. This is strong evidence supporting the degradation mechanism proposed in Figure 1 (a). 
Finally, when using i-propanol as the solvent, the o-PBE mass spectrum presented some discrepancies compared to its linear counterpart (Figure 2). During its fragmentation, the 2[(propan-2-yloxy)methyl] phenol mechanically undergoes pathway 1 with the production of $m / z$ 51,78 and 106. However, due to the absence of $m / z 121$ and the presence of $m / z 43$ and 124 instead, it is likely that instead of pathway 2 , these ions undergo an onium reaction where the alkyl linked to the charged heteroatom. In this case the oxonium ion is cleaved with a transfer of a hydrogen from the alkyl substituent to the heteroatom leading to propylium ions $(\mathrm{m} / \mathrm{z} 43)$ and 2-(hydroxymethyl)phenol ions ( $\mathrm{m} / \mathrm{z}$ 124) (Figure 1 (c)). Proposing the exact reason for this mechanical change in the fragmentation would be speculative because the precise origin of the hydrogen that is transferred is not known in such type of reaction, however they have been observed in ethers decomposition sequences [13].

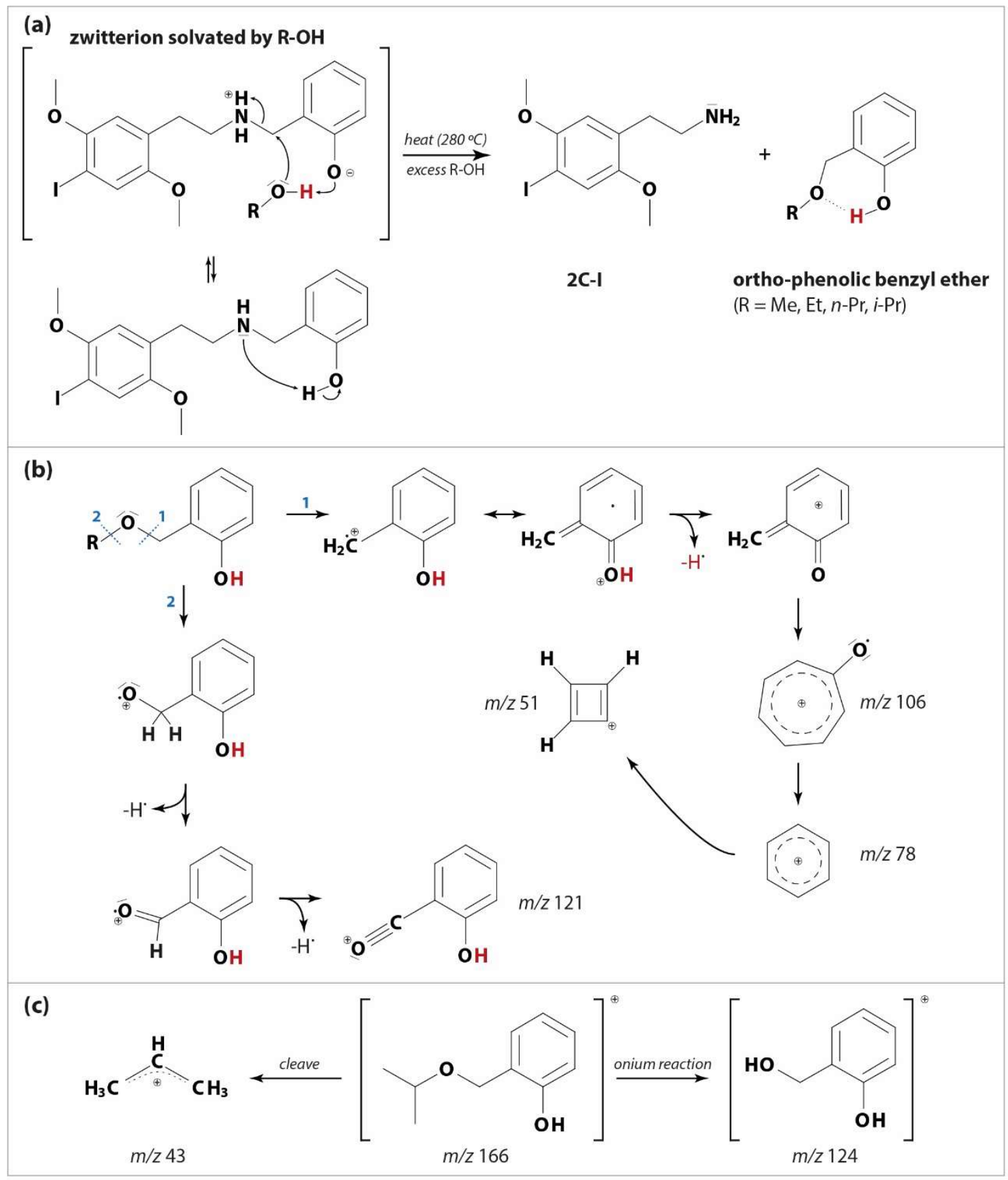


Fig 1 (a)Thermal degradation of 25I-NBOH mechanism and production of 2C-I and o-PBEs; (b) $o-\mathrm{PBEs} \mathrm{El}^{+}$fragmentation pathways $(\mathrm{R}=\mathrm{Me}, \mathrm{Et}, n-\mathrm{Pr})$; (c) 2-[(propan-2-yloxy)methyl]phenol $\mathrm{El}^{+}$ fragmentation (o-PBE created with $i$-propanol as the solvent)

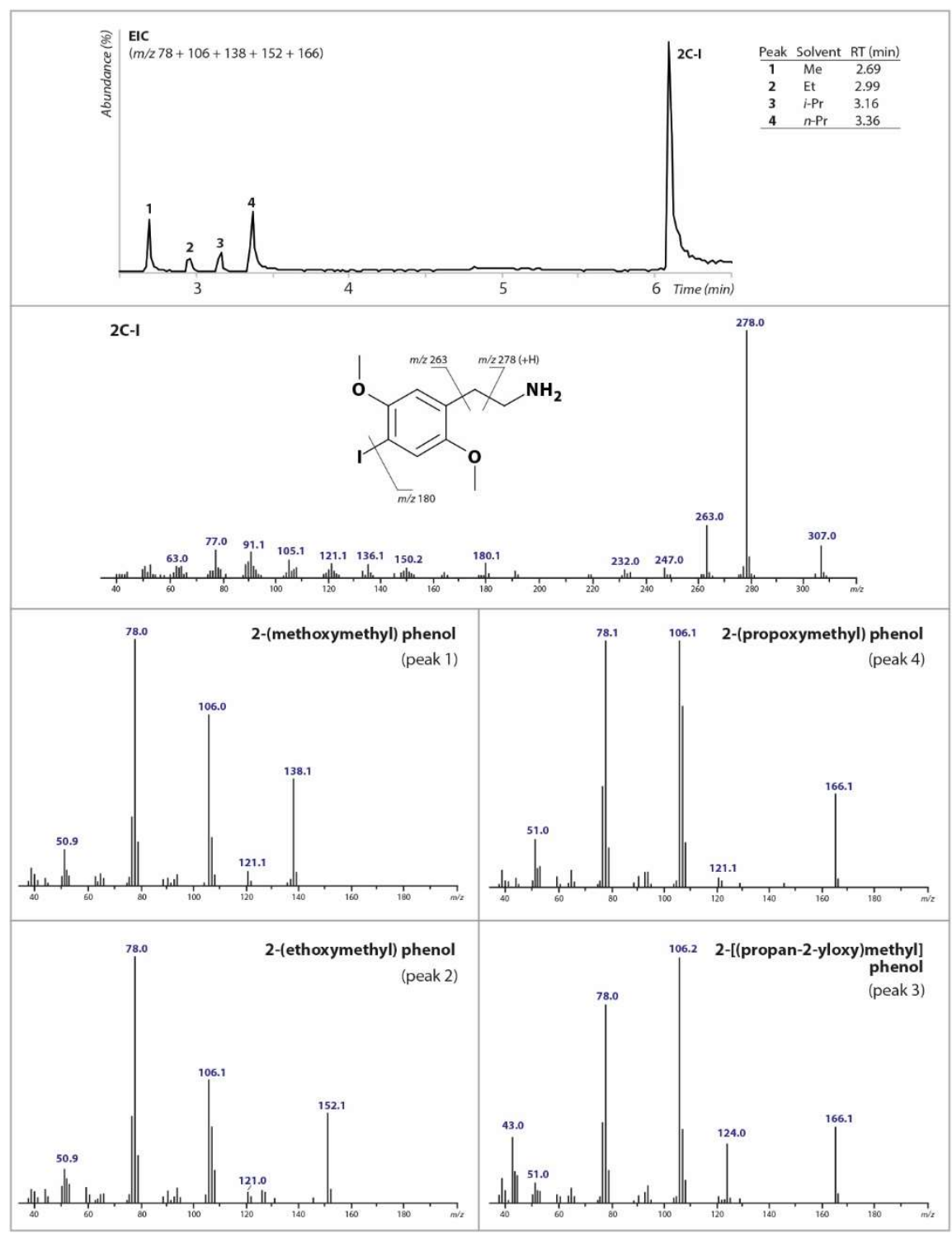

Fig $2 \mathrm{EI}^{+}$extracted ion chromatogram (EIC) $(m / z 78,106,138,152$, and 166) obtained when analysing $25 \mathrm{I}-\mathrm{NBOH}$ with a mix solvent (Me, Et, $n-\mathrm{Pr}, i-\mathrm{Pr}$ ) and corresponding respective mass spectra of the $2 \mathrm{C}-\mathrm{I}$ and the $o$-PBEs created.

\section{Method Adjustments}

In order to avoid the 25I-NBOH fragmentation into 2-(methoxymethyl)phenol (peak 1) and 2C-I (peak 2), several methods' modifications were experimented. Adjustments in the injector temperature were the first parameter optimized. Assuming that a thermal degradation occurred in the injector (due to sharp peak shape), temperature was lowered from $280^{\circ} \mathrm{C}$ to $170^{\circ} \mathrm{C}$. The lower the injector temperature, the more difficult it is for the compound to vaporize and a lower signal for peak 2 was achieved, confirming the results previously described by Coelho Neto el at 
[8]. The 25I-NBOH molecular peak was not observed in any chromatogram. The peaks behavior associated to injector temperature changes can be observed in Figure 3.

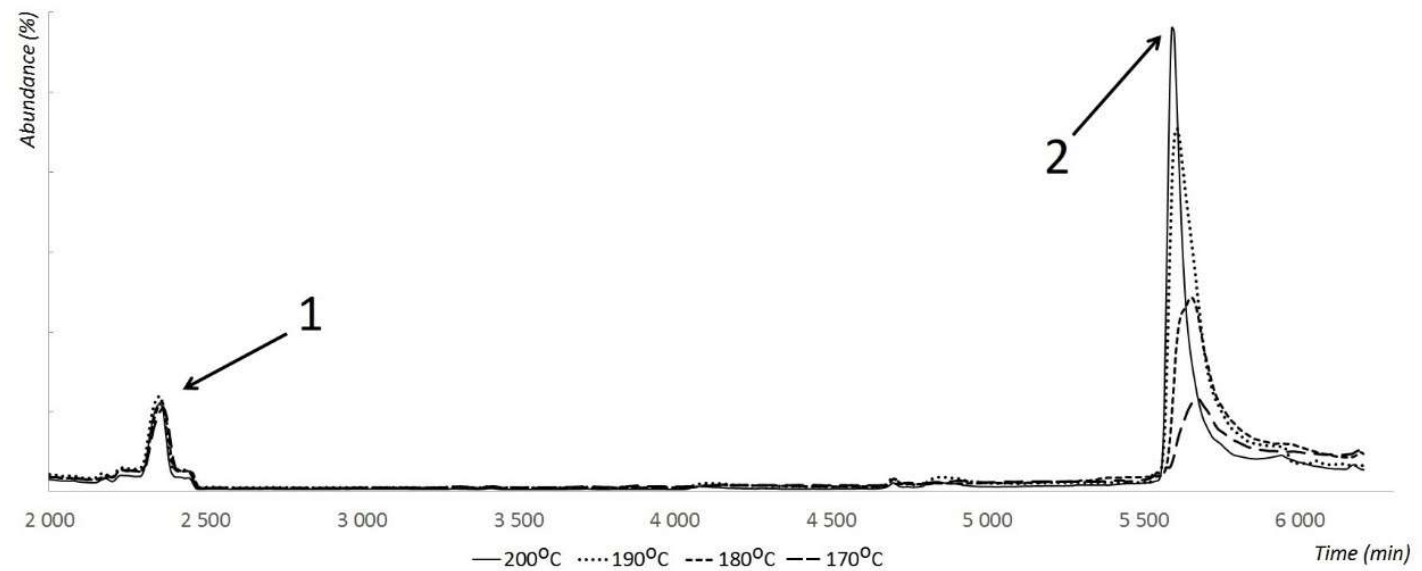

Fig 3 Injector temperature influence on peaks 1 and 2 on a 25I-NBOH GC analysis.

Secondly, split ratio was adapted to maximize the possibility of occurrence of $25 \mathrm{I}-\mathrm{NBOH}$ molecular ion in the chromatography results. Split (50:1, 25:1, 10:1) and splitless injections were tested. The best abundance detected on peaks 1 and 2 was using split ratio 10:1, nonetheless no molecular peak was recognized on those runs. Those results are in agreement with the ones previous reported [8].

In an attempt to reduce elution temperature and reduce the prospect thermal degradation experiments with different flows rate $(0.6$ to $1.5 \mathrm{~mL} / \mathrm{min})$ were conducted. For the smaller fragment (peak 1), flow rate of $0.8 \mathrm{~mL} / \mathrm{min}$ culminate in better peak abundance. Although for peak 2 , flow rate of $1.2 \mathrm{~mL} / \mathrm{min}$ presented better results.

\section{Thermal Analysis}

Despite all the efforts and experiments carried out, the 25I-NBOH molecular ion has not been detected as a result of the mere change in the chromatographic conditions. Thermogravimetric Analysis (TGA) and Differential Scanning Calorimetry (DSC) analysis were carried out with the aim of understanding the thermal behavior of $25 \mathrm{I}-\mathrm{NBOH}$ in comparison with its less polar counterpart 25I-NBOMe.

Thermal curves, as determined by DSC, are shown in Figure 4(a) For both compounds, 25INBOMe and 25I-NBOH. The DSC curves displayed one major endothermic region corresponding to fusion peaks $\left(167^{\circ} \mathrm{C}-25 \mathrm{I}-\mathrm{NBOMe} ; 203^{\circ} \mathrm{C}-25 \mathrm{I}-\mathrm{NBOH}\right)$ and one exothermic region corresponding to a common decomposition peak ( $265^{\circ} \mathrm{C}-25 \mathrm{I}-\mathrm{NBOMe}$ and $\left.25 \mathrm{I}-\mathrm{NBOH}\right)$. The shapes of 25I-NBOMe and 25I-NBOH decomposition peaks are very distinct with $25 \mathrm{I}-\mathrm{NBOH}$ peak more asymmetric with a prominent fronting pattern. Comparing both compounds, a difference of almost $40^{\circ} \mathrm{C}$ between fusion points was registered. This difference demonstrates that $25 \mathrm{I}-$ $\mathrm{NBOH}$ has a smaller temperature window between fusion and decomposition directly reflecting on its thermal stability. The 25I-NBOH DSC thermogram shows that as soon as the compound reaches fusion it will start decomposing supporting the fact that only degradation products may be seen in GC-MS analysis. 
TGA thermograms, shown on Figure 4(b), corroborate the occurrence for both compounds of a common thermal decomposition at approximately $265^{\circ} \mathrm{C}$, which is evidenced by a significant sample mass loss.
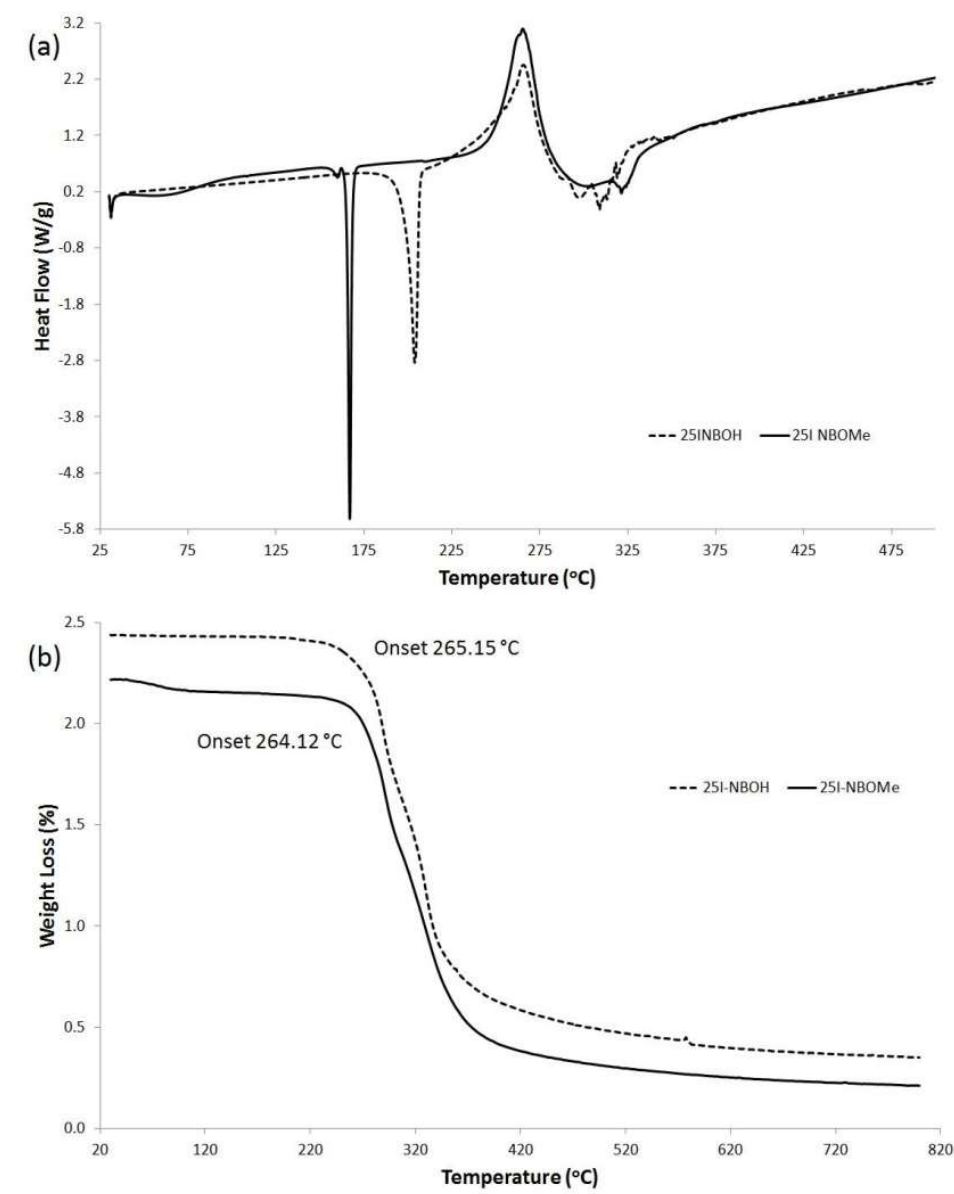

Fig 4 Thermal analysis for 25I-NBOH and 25I-NBOMe. (a) Differential Scanning Calorimetry (DSC) of 25I-NBOH and 25I-NBOMe. (b) Thermogravimetric Analysis (TGA) of 25I-NBOH and 25INBOMe.

\section{Derivatization}

Thermal analysis results indicated that no GC method adjustment would be capable of unequivocally identifying 25I-NBOH. Consequently, derivatization was investigated as the next approach to overcome 25I-NBOH thermal degradation. A series of experiments using three different derivatization agents (MSTFA, BSTFA, and acetic anhydride) was performed.

MSTFA silylation reaction generated two derivatives: mono-TMS and di-TMS derivatives, which followed the expected order of elution for such species with the mono-TMS version eluting slightly ahead of its di-TMS counterpart (RT $=7.89 \mathrm{~min}$ and $7.98 \mathrm{~min}$ respectively) (Figure 5). Mono-TMs was produced when MSTFA reacted just with the -OH present in the 25I-NBOH molecule. This fact was confirmed as $\mathrm{m} / \mathrm{z}=179$ is formed by heterolytic cleavage at the amino group resulting in a highly stable tropilic ion with the $\mathrm{Si}(\mathrm{CH} 3) 3$ group attached to the $\mathrm{OH}$ in the molecule (Figure 5). Di-TMS is generated when a second derivatization reaction takes place at the $-\mathrm{NH}$ site. Distinctly, using BSTFA derivatization only mono-TMS derivative is visualized 
(Figure 5). Besides the production of two compounds in the derivatization process, higher chromatographic peak abundances indicate MSTFA as a more effective derivatization reagent than BSTFA.

On the other derivatization experiment, acetic anhydride was used to produce an acylation reaction as it is known to improve the stability of compounds that are thermally labile by inserting protecting groups into the molecule[15]. The acylation promoted the introduction of two acyl groups on both active hydrogen $(-\mathrm{OH}$ and $-\mathrm{NH})$ and yielded high intensity di-acylated derivative chromatograms (Figure 5). As in the case of MSTFA the use of acylation indicated the presence of glucose (or any of its stereoisomers) in some of the blotter paper samples with a good match (926 out of 1000) for its pentaacetate form in the NIST 11 mass spectral library. It is possible that sugar has been added on blotter papers with the intent of hiding the alleged bitter taste of 25I-NBOH previously reported in user forums[16-18].

It can be noted that the typical 2C-I peak found in underivatized run (RT 6.16) was not observed with the derivatized run. This is indication that the use of derivatization reagent in excess and lengthy incubation time enabled completion of the derivatization process and that the molecule was "stabilized" through the process, enabling unequivocal identification. 

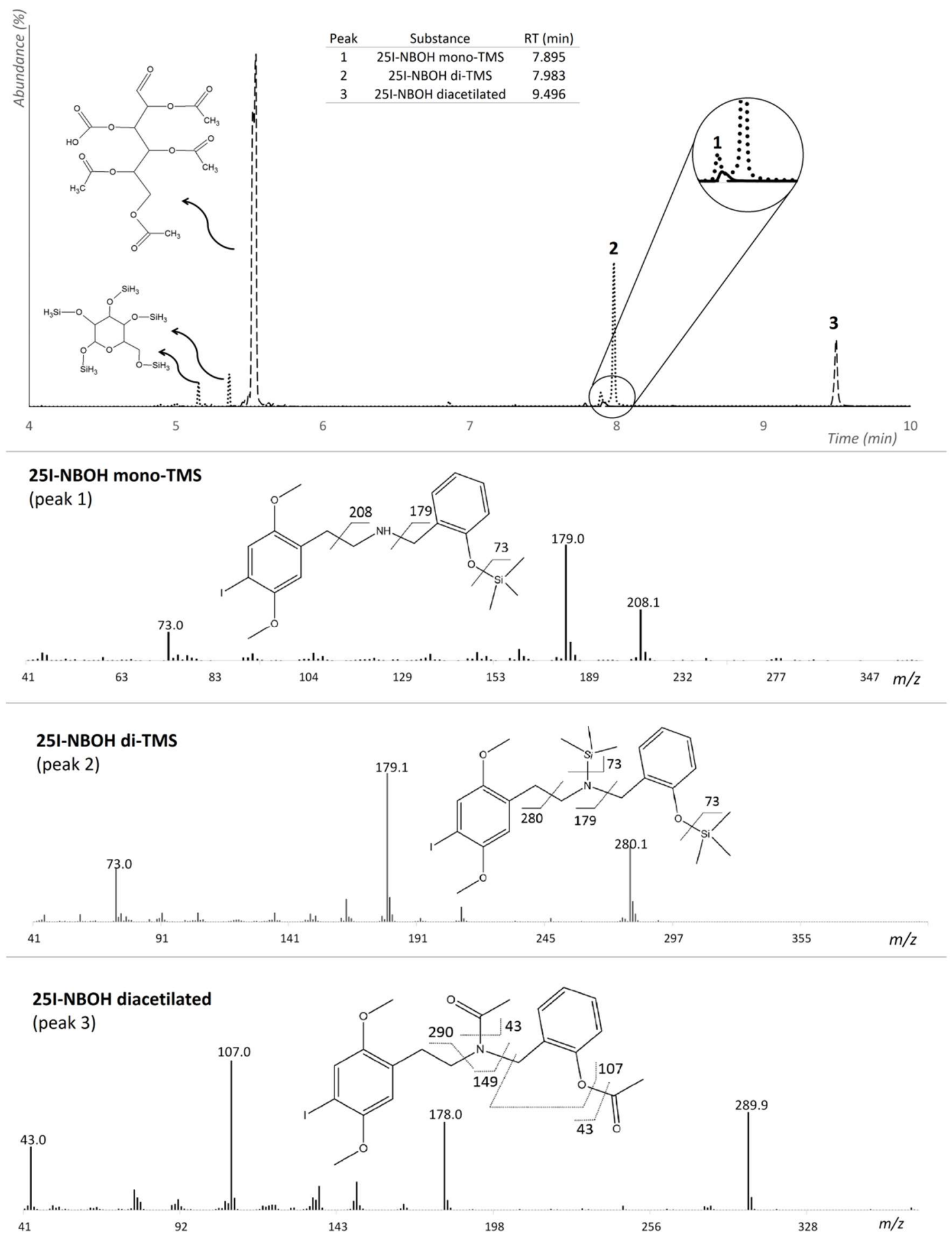

Fig 5 GC-MS profiles and molecular structure obtained after chemical derivatization of 25INBOh with MSTFA (…...), BSTFA (-) and acetic anhydride (------).

\section{Conclusion}

In this paper, we provided a detailed study of the 25I-NBOH thermal behavior. Degradation and fragmentation pathways were presented and deciphered while DSC and TGA analysis demonstrated that prompt decomposing occurs when the compound reaches fusion point. 
Despite all the effort adjusting the GC-MS methods, 25I-NBOH degradation in the injector was unavoidable. However, derivatization enabled the stabilization of the molecule and identification could be successfully made via both silylated and acylated derivatives. This indicates how GC-MS can be used to provide correct identification for this class of compounds.

Finally, forensic experts need to be aware of these cases in order to implement non-thermal alternative analytical techniques and chemical derivatization in order to correctly identify the ever-emerging new classes of NPS.

\section{Acknowledgements}

The authors are thankful to Instituto de Criminalıstica da Polıcia Civil do Distrito Federal (PCDF), Instituto Nacional de Criminalística (INC), Fundação de Amparo a Pesquisa do Distrito Federal (FAPDF), Fundação de Peritos em Criminalıstica llaraine Acacio Arce (FPCIAA) and University of Lincoln for providing the samples used in this study and for technical and financial support.

\section{Compliance with ethical standards}

Conflict of interest Authors declare no conflicts of interest.

Ethical approval This article does not contain any studies with human participants or animals performed by any of the authors.

\section{References}

1. United Nations Office on Drugs and Crime (2017) Global synthetic drugs assessment: Amphetamine-type stimulants and new psychoactive substances. 81 . https://doi.org/10.18356/19d5a8d1-en

2. United Nations Office on Drugs and Crime (2018) Understanding the synthetic drug market: the NPS factor. Glob Smart Updat 19:1-12

3. European Monitoring Centre for Drugs and Drug Addiction (EMCDDA) (2018) European Drug Report: Trends and Developments

4. Arantes LC, Júnior EF, de Souza LF, et al (2017) 25I-NBOH: a new potent serotonin 5HT2A receptor agonist identified in blotter paper seizures in Brazil. Forensic Toxicol 35:408-414. https://doi.org/10.1007/s11419-017-0357-x

5. Clas SD, Dalton CR, Hancock BC (1999) Differential scanning calorimetry: Applications in drug development. Pharm Sci Technol Today 2:311-320.

https://doi.org/10.1016/S1461-5347(99)00181-9

6. Chiu M, Prenner E (2011) Differential scanning calorimetry: An invaluable tool for a detailed thermodynamic characterization of macromolecules and their interactions. J Pharm Bioallied Sci 3:39-59. https://doi.org/10.4103/0975-7406.76463

7. Coats AW, Redfern JP (1963) Thermogravimetric Analysis A Review. Analyst 88:906-924

8. Coelho Neto J, Andrade AFB, Lordeiro RA, et al (2017) Preventing misidentification of 25I-NBOH as 2C-I on routine GC - MS analyses. Forensic Toxicol 35:415-420. 
https://doi.org/10.1007/s11419-017-0362-0

9. Belchior de Andrade AF, Gonzalez-Rodriguez J (2019) Electroanalytical identification of 25I-NBOH and 2C-I via differential pulse voltammetry: a rapid and sensitive screening method to avoid misidentification. Analyst 144:2965-2972.

https://doi.org/10.1039/c9an00062c

10. Magalhães L de O, Arantes LC, Braga JWB (2019) Identification of NBOMe and NBOH in blotter papers using a handheld NIR spectrometer and chemometric methods.

Microchem J 144:151-158. https://doi.org/10.1016/j.microc.2018.08.051

11. de Morais DR, Barbosa IL, Cunha KF, et al (2017) EASI-IMS an expedite and secure technique to screen for 25I-NBOH in blotter papers. J Mass Spectrom 52:701-706. https://doi.org/10.1002/jms.3977

12. United Nations Office on Drugs and Crime. (2018) INTERNATIONAL QUALITY ASSURANCE PROGRAMME (IQAP) - INTERNATIONAL COLLABORATIVE EXERCISES (ICE): Summary Report. 103

13. Meier H, Zeeh B, Hesse M (2008) Spectroscopic Methods in Organic Chemistry, 2nd ed. Thieme Medical Publishers Inc, New York

14. Radeglia R (1981) Hesse, M., Meier, H. und Zeeh, B.: Spektroskopische Methoden in der organischen Chemie. VI, 478 S. 169 Abb., 86 Tab., $12 \times 19$ cm. Stuttgart: Georg Thieme Verlag 1979. (Thieme Taschenlehrbuch der organischen Chemie, A Theoretische und allgemeine Gebiete, B. J für Prakt Chemie 323:175-175. https://doi.org/10.1002/prac.19813230126

15. Orata F (2012) Derivatization Reactions and Reagents for Gas Chromatography Analysis. In: Ali Mohd M (ed) Advanced Gas Chromatography - Progress in Agricultural, Biomedical and Industrial Applications. IntechOpen, Rijeka

16. 25I-NBOH. http://nervewing.blogspot.com/2014/05/25i-nboh.html. Accessed 5 Jan 2020

17. Psychonautwiki 25I-NBOH. https://psychonautwiki.org/wiki/25I-NBOH. Accessed 5 Jan 2020

18. Hulu Nice and Easy 25I-NBOH. https://erowid.org/experiences/exp.php?ID=107750. Accessed 5 Jan 2020 